\title{
A European benchmarking system to evaluate in-hospital mortality rates in acute coronary syndrome: The EURHOBOP project ${ }^{\text {th }}$
}

\author{
Irene R. Dégano ${ }^{\mathrm{a}, 1}$, Isaac Subirana a,b,1, Marina Torre ${ }^{\mathrm{c}}$, María Grau ${ }^{\mathrm{a}}$, Joan Vila a,b ${ }^{\mathrm{a}}$, Danilo Fusco ${ }^{\mathrm{d}}$, \\ Inge Kirchberger ${ }^{e, f}$, Jean Ferrières ${ }^{g}$, Antti Malmivaara ${ }^{h}$, Ana Azevedo ${ }^{i}$, Christa Meisinger ${ }^{e, f}$, Vanina Bongard ${ }^{j}$, \\ Dimitros Farmakis ${ }^{\mathrm{k}}$, Marina Davoli ${ }^{\mathrm{d}}$, Unto Häkkinen ${ }^{\mathrm{h}}$, Carla Araújo ${ }^{\mathrm{i}}$, John Lekakis ${ }^{\mathrm{k}}$, Roberto Elosua ${ }^{\mathrm{a}}$, \\ Jaume Marrugat ${ }^{\mathrm{a}, *}$, on behalf of the EURHOBOP investigators \\ a REGICOR Study Group, Cardiovascular Epidemiology and Genetics Group, IMIM, Hospital del Mar Medical Research Institute, Barcelona, Spain \\ b CIBER Epidemiology and Public Health, Spain \\ ' Istituto Superiore di Sanità, Rome, Italy \\ d Dipartimento di Epidemiologia ASL RME, Rome, Italy \\ e MONICA/KORA Myocardial Infarction Registry, Augsburg, Germany \\ ${ }^{\mathrm{f}}$ Helmholtz Zentrum München, German Research Center for Environmental Health, Institute for Epidemiology II, Neuherberg, Germany \\ ${ }^{g}$ Department of Cardiology, Toulouse Rangueil Hospital, Toulouse University School of Medicine, Toulouse, France \\ ${ }^{\text {h }}$ Centre for Health and Social Economics, Helsinki, Finland \\ i Department of Clinical Epidemiology, Predictive Medicine and Public Health, University of Porto Medical School, Institute of Public Health of the University of Porto (ISPUP), Porto, Portugal \\ j Department of Epidemiology, UMR 1027 INSERM, Toulouse University, Toulouse, France \\ k ATTIKON Hospital, University of Athens Medical School, Athens, Greece
}

\section{A R T I C L E I N F O}

\section{Article history:}

Received 27 August 2014

Received in revised form 30 December 2014

Accepted 4 January 2015

Available online 7 January 2015

\section{Keywords:}

Benchmarking

Hospital performance

Acute myocardial infarction

Percutaneous coronary intervention

In-hospital mortality

\begin{abstract}
A B S T R A C T
Background: Hospital performance models in acute myocardial infarction (AMI) are useful to assess patient management. While models are available for individual countries, mainly US, cross-European performance models are lacking. Thus, we aimed to develop a system to benchmark European hospitals in AMI and percutaneous coronary intervention (PCI), based on predicted in-hospital mortality.

Methods and results: We used the EURopean HOspital Benchmarking by Outcomes in ACS Processes (EURHOBOP) cohort to develop the models, which included 11,631 AMI patients and 8276 acute coronary syndrome (ACS) patients who underwent PCI. Models were validated with a cohort of 55,955 European ACS patients. Multilevel logistic regression was used to predict in-hospital mortality in European hospitals for AMI and PCI. Administrative and clinical models were constructed with patient- and hospital-level covariates, as well as hospital- and country-based random effects. Internal cross-validation and external validation showed good discrimination at the patient level and good calibration at the hospital level, based on the C-index (0.736-0.819) and the concordance correlation coefficient (55.4\%-80.3\%). Mortality ratios (MRs) showed excellent concordance between administrative and clinical models (97.5\% for AMI and 91.6\% for PCI). Exclusion of transfers and hospital stays $\leq 1$ day did not affect in-hospital mortality prediction in sensitivity analyses, as shown by MR concordance (80.9\%-85.4\%). Models were used to develop a benchmarking system to compare in-hospital mortality rates of European hospitals with similar characteristics.

Conclusions: The developed system, based on the EURHOBOP models, is a simple and reliable tool to compare inhospital mortality rates between European hospitals in AMI and PCI.
\end{abstract}

(C) 2015 Elsevier Ireland Ltd. All rights reserved.

\section{Introduction}

Acute coronary syndrome (ACS) is the leading cause of morbimortality in Europe, emphasising the need of assessing ACS quality

\footnotetext{
All authors take responsibility for all aspects of the reliability and freedom from bias of the data presented and their discussed interpretation.

* Corresponding author at: Carrer Dr Aiguader 88, E-08003 Barcelona, Spain.

E-mail address: jmarrugat@imim.es (J. Marrugat).

${ }^{1}$ Both authors have equally contributed to this manuscript.
}

of care [1]. To assess quality of care in ACS, it would be useful to analyse hospital performance based on quality indicators; such as structure (e.g. access to cardiovascular specialists), process (e.g. timely and appropriate reperfusion) and outcomes (e.g. death) [2]. Compared to structure and process, outcome indicators provide a more global measure of quality of care and are the most relevant to patients and physicians [2,3]. In addition, mortality - the most widely used outcome - has been recommended as a key indicator of the quality of acute myocardial infarction (AMI) management [4]. 
In the last decade, several models have been developed to analyse hospital performance in ACS management based on in-hospital or 30day mortality [5-10]. Krumholz et al. provided the first model to estimate AMI 30-day mortality for benchmarking purposes, using administrative data [5]. Following similar methodology, other authors developed models to estimate AMI [6-8], ACS [9], and percutaneous coronary intervention (PCI) [10] mortality, mainly using administrative data.

The usefulness of hospital performance models for quality of care improvement is based on the actions that would be implemented as a result of the information obtained. For example, it would be expected that a hospital/department whose AMI mortality is higher than the mortality observed in other units with similar characteristics, regarding patients and resources, would revise and improve their current AMI protocols. Thus, European hospitals, cardiovascular departments and coronary units must be able to compare themselves to others with similar characteristics. For this comparison to be reliable, the data used to develop the models should be representative of European hospitals. However, the available models were mainly developed in the US [5,7, $8,10]$ and most of them, including the only PCI model, were developed for patients older than 64 years [5,8,10]. In addition, cross-European performance models, which would be representative of European hospitals and would allow a common strategy for ACS quality of care improvement in Europe, are lacking. To fill this gap, we designed the EURopean HOspital Benchmarking by Outcomes in ACS Processes (EURHOBOP) project [11]. The EURHOBOP, was a cohort study funded by the European Commission, designed to provide a valid ACS benchmarking system to the European Community. The goal of this system was to allow cardiologists and other professionals from European hospitals to easily monitor their outcomes in key ACS diagnosis and procedures.

The aim of the present study was to derivate and validate a set of models to benchmark in-hospital mortality rates in European hospitals, in $\mathrm{AMI}$ and $\mathrm{PCI}$, taking into account the country as well as hospital and patient characteristics. The secondary aim was to provide a computerbased tool, using the developed models, to allow cardiologists to compare the data from their own practice with others.

\section{Methods}

The EURHOBOP Study complies with the Declaration of Helsinki and was approved by the ethics committee of the Parc de Salut Mar (IMIM-PSMAR 2010/3779/I) of Barcelona, Spain.

\subsection{Data collection}

The EURHOBOP cohort included 15,170 ACS patients from 68 hospitals located in: Finland, France, Germany, Greece, Italy, Portugal, and Spain. Data collection in the EURHOBOP study has been previously described [12]. Briefly, at least 2000 patients were retrospectively recruited per country from 2008 to 2012, with a mean of 221 consecutive patients recruited per hospital. Patient- and hospital-level data were obtained from the letter of discharge and from medical records (whether on paper or electronic). Patient characteristics included demographic variables, cardiovascular (CV) risk factors, comorbidities, procedures and complications during hospitalization, and admission and discharge data. Hospital characteristics included the number of beds and cardiology patients discharged, as well as ACS care facilities. Collected variables are listed in the Supplementary material online, Table S1.

\subsection{Study population and outcome}

EURHOBOP patients with a discharge diagnosis of unstable angina (UA) or AMI (International Classification of Diseases 10th revision: I21.0-I21.9 and I20.0) were selected for the present study. Two patient subsets were chosen for hospital benchmarking due to their importance in ACS management: patients with a discharge diagnosis of AMI and patients who underwent $\mathrm{PCI}$ during the index admission. Patients from hospitals that recruited less than 10 patients for any of the subsets were excluded from the analysis. The flowchart of the included patients is presented in Fig. S1. The selected outcome was inhospital mortality from any cause during the index admission.

\subsection{Candidate variables}

Variables predictive of in-hospital mortality were chosen based on literature evidence and clinical experience. Candidate variables for analysis were selected on the basis of the reference time (defined as admission), as suggested for models reporting health outcomes [13]. Thirteen candidate variables remained for the analysis: 8 at the patient level (age sex, hypertension, diabetes, past history of CV diseases, ACS type, presence of cardiogenic shock or acute pulmonary oedema on admission and presence of renal failure on admission), and 5 at the hospital level (presence of intensive care unit (ICU), coronary care unit (CCU), catheterization laboratory, cardiac surgery, and university hospital status). Two sets of variables were defined: a clinical set, containing all candidate variables, and an administrative set, excluding the presence of cardiogenic shock or acute pulmonary oedema and the presence of renal failure on admission.

\subsection{Model description}

Two hierarchical logistic mixed models, one with the clinical and the other with the administrative set of variables, were fitted to each patient subset (AMI or PCI). Inhospital mortality was the binary outcome variable; patient and hospital characteristics constituted the fixed-effect explanatory variables. Hospital and country were also included as random-effect variables to deal with the hierarchical clustering of data.

\subsection{Expected in-hospital mortality calculation}

To calculate the expected in-hospital mortality the model was fitted using all patients except those admitted to the hospital for which the expected in-hospital mortality was being computed. This procedure, also known as cross-validation was repeated for all hospitals. Two types of expected in-hospital mortality rates were computed: (i) for hospitals located in countries included in the sample, by including the specific country effect in the model, and (ii) for hospitals from other countries, using the same hospitals data but assuming that they were from a country not included in the sample and setting the country effect to zero. Methodological detail regarding the computation of expected in-hospital mortality rates is provided in the Supplementary material online.

Table 1

Comparison of patients from the derivation and the validation cohort.

\begin{tabular}{|c|c|c|c|c|c|c|}
\hline & \multicolumn{3}{|l|}{ AMI } & \multicolumn{3}{|l|}{ PCI } \\
\hline & \multirow{2}{*}{$\begin{array}{l}\text { Validation } \\
\mathrm{N}=54,083\end{array}$} & \multirow{2}{*}{$\begin{array}{l}\text { Derivation } \\
\mathrm{N}=11,631\end{array}$} & \multirow[t]{2}{*}{ Overall p-value } & \multirow{2}{*}{$\begin{array}{l}\text { Validation } \\
\mathrm{N}=25,747\end{array}$} & \multirow{2}{*}{$\begin{array}{l}\text { Derivation } \\
\mathrm{N}=8276\end{array}$} & \multirow[t]{2}{*}{ Overall p-value } \\
\hline & & & & & & \\
\hline Age; mean (SD) & $69.0(13.4)$ & $67.7(13.6)$ & $<0.001$ & $65.2(12.5)$ & $64.6(12.6)$ & $<0.001$ \\
\hline Sex: Female & $17,972(33.2 \%)$ & $3599(30.9 \%)$ & $<0.001$ & $6500(25.2 \%)$ & $2050(24.8 \%)$ & 0.392 \\
\hline Hypertension & $14,015(25.9 \%)$ & $6100(52.4 \%)$ & 0.000 & $6336(24.6 \%)$ & $4364(52.7 \%)$ & 0.000 \\
\hline Diabetes & $7856(14.5 \%)$ & $2904(25.0 \%)$ & $<0.001$ & $3139(12.2 \%)$ & $1841(22.2 \%)$ & $<0.001$ \\
\hline Past history of CVD & $14,348(26.5 \%)$ & $3819(32.8 \%)$ & $<0.001$ & $6018(23.4 \%)$ & $2679(32.4 \%)$ & $<0.001$ \\
\hline ACS & & & $<0.001$ & & & $<0.001$ \\
\hline Non-STEACS & $28,033(52.2 \%)$ & $5366(46.6 \%)$ & & $10,696(41.8 \%)$ & $3848(47.0 \%)$ & \\
\hline STEACS & $23,643(44.0 \%)$ & $5530(48.1 \%)$ & & $14,505(56.6 \%)$ & $4002(48.8 \%)$ & \\
\hline Non classifiable & $2074(3.86 \%)$ & $607(5.28 \%)$ & & $409(1.60 \%)$ & $343(4.19 \%)$ & \\
\hline Shock/pulmonary oedema & $1905(3.53 \%)$ & $831(7.14 \%)$ & $<0.001$ & $695(2.70 \%)$ & $400(4.83 \%)$ & $<0.001$ \\
\hline Renal failure & $4150(8.28 \%)$ & $1197(10.3 \%)$ & $<0.001$ & $1274(5.54 \%)$ & $543(6.56 \%)$ & 0.001 \\
\hline In-hospital mortality & $4469(8.26 \%)$ & $868(7.46 \%)$ & 0.004 & 977 (3.79\%) & $278(3.36 \%)$ & 0.073 \\
\hline
\end{tabular}

Unless indicated represented values are: frequencies (percentages).

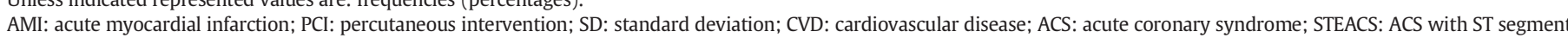
elevation. 
Table 2

Characteristics of the participating hospitals in the derivation cohort.

\begin{tabular}{lllllll}
\hline & ICU & CCU & Cath Lab & Cardiac & University & Total \\
\hline ICU: intensive care unit & 61 & 55 & 54 & 32 & 29 & 61 \\
CCU: coronary care unit & 55 & 59 & 54 & 33 & 29 & 59 \\
Cath Lab: catheterization & 54 & 54 & 55 & 33 & 29 & 55 \\
$\quad$ laboratory & & & & & & \\
Cardiac: cardiac surgery & 32 & 33 & 33 & 33 & 25 & 33 \\
University: university hospital & 29 & 29 & 29 & 25 & 30 & 30 \\
\hline
\end{tabular}

Pairwise combinations and marginal frequencies for the included hospital characteristics in the 68 participating hospitals.

\subsection{Variable selection}

A forward stepwise procedure was used to select the variables included in the model from the list of candidate variables. The selection was based on the maximization of the concordance correlation coefficient (CCC). The CCC was computed as the agreement between observed and expected in-hospital mortality rates for each hospital, weighted for the number of patients recruited by that hospital. Expected and observed mortality rates were normalized with the squared-sinus transformation prior to CCC calculation. The step-forward process was repeated until all candidate variables were included (Fig. S2). An extra step was conducted to remove the variables that did not improve the CCC and were not significantly associated with the outcome. Sex was forced into the AMI models to account for the demographic and clinical differences between men and women with an AMI. Due to the high co-linearity of the ICU, CCU and catheterization laboratory variables, if one of these entered in the model, the other two were removed from the list of candidate variables.

\subsection{Model validation}

Model validation was assessed internally and externally using two measures: the Cindex, to assess model discrimination at the patient level, and the CCC between observed and expected rates, to assess model calibration at the hospital level. The CCC was provided together with its $95 \%$ confidence interval and an "upper reference value", which was calculated under the hypothesis that, with our sample size, the variables explained $100 \%$ of the observed variability. Internal validation was performed by cross-validation as described in Section 2.5. External validation was performed using an independent cohort of 55,955 ACS patients who were included in 3 European registries: the Cooperative Health Research in the Region of Augsburg (KORA) Myocardial Infarction Registry (2005-2011, $\mathrm{n}=3973$ ) [14], the Lazio Region Hospital Information System (2008-2013, $\mathrm{n}=44,855$ ) [15] and the Spanish MASCARA ACS Study (2004-2005, $\mathrm{n}=$ 7127) [16]. Hospitals with less than 10 patients in any of the analysed subsets were excluded.
2.8. Concordance between administrative and clinical models

Administrative and clinical models were compared by assessing the concordance of mortality ratios (MRs) $[5,10]$ for each model type. MRs were computed as the ratio of predicted and expected in-hospital mortality rates in each hospital. The predicted in-hospital mortality rates were calculated as the expected rates, described in Section 2.5, but adding the specific effect of each hospital. MRs were log-transformed for normalization. Log-MRs from administrative and clinical models were compared for each patient subset (AMI and $\mathrm{PCI}$ ) using the CCC statistic. Methodological detail regarding the computation of predicted in-hospital mortality rates is provided in the Supplementary material online.

\subsection{Sensitivity analyses}

The four described models were re-fitted on a subsample, excluding transferred patients and patients who stayed $\leq 1$ day at the hospital. The flowchart of the included patients is presented in Fig. S3. Cross-validation was performed with the C-index and CCC measures. MRs were calculated for each model as described and log-MRs were compared to the log-MRs from the full sample using the CCC.

Statistical analysis was performed with R Statistical Package (R Foundation for Statistical Computing, Vienna, Austria; Version 3.0.2) [17].

\section{Results}

\subsection{Patient and hospital characteristics}

In the derivation cohort patients had a mean age of 66 years and $28 \%$ were women. Patient characteristics by subset are presented in Table 1. Crude in-hospital mortality was 7.5\% in AMI patients and 3.4\% in the patients who underwent PCI. From the included cases, $25.7 \%$ and $19.6 \%$ involved hospital transfers in the AMI and the PCI subset, respectively. The proportion of patients discharged within the first $24 \mathrm{~h}$ was $8.2 \%$ in the AMI subset and $5.5 \%$ in the PCI subset.

Regarding hospital characteristics, more than $75 \%$ of the hospitals had ICU, CCU, or catheterization laboratory, or a combination of these facilities. Around $50 \%$ of the hospitals had cardiac surgery facilities or were university hospitals (Table 2 ).

\subsection{Model derivation}

The administrative and clinical AMI models included the following predictor variables: sex, age, hypertension, diabetes, ACS type, and

Table 3

Derivation and validation of administrative and clinical models.

\begin{tabular}{|c|c|c|c|c|}
\hline & \multicolumn{2}{|l|}{ AMI } & \multicolumn{2}{|l|}{$\mathrm{PCI}$} \\
\hline & $\begin{array}{l}\text { Clinical } \\
\mathrm{N}=11,503\end{array}$ & $\begin{array}{l}\text { Administrative } \\
\mathrm{N}=11,503\end{array}$ & $\begin{array}{l}\text { Clinical } \\
\mathrm{N}=8192\end{array}$ & $\begin{array}{l}\text { Administrative } \\
\mathrm{N}=8192\end{array}$ \\
\hline \multicolumn{5}{|l|}{ Included variables OR [95\% CI] } \\
\hline Sex: Female & $0.98[0.83-1.15]$ & $1.04[0.88-1.21]$ & & \\
\hline Age & $1.07[1.06-1.08]$ & $1.07[1.06-1.08]$ & & 1.07 [1.05-1.08] \\
\hline Hypertension & $0.65[0.55-0.78]$ & $0.69[0.58-0.82]$ & & 0.77 [0.57-1.03] \\
\hline Diabetes & 1.30 [1.08-1.55] & $1.45[1.22-1.72]$ & $1.26[0.92-1.72]$ & $1.37[1.02-1.85]$ \\
\hline Past history of CVD & & $1.14[0.97-1.34]$ & $1.56[1.17-2.06]$ & $1.31[1.00-1.73]$ \\
\hline ACS: STEACS & $3.23[2.70-3.86]$ & $3.00[2.52-3.56]$ & $5.89[4.17-8.31]$ & $6.50[4.66-9.06]$ \\
\hline ACS: Non classifiable & $2.73[2.05-3.62]$ & 2.99 [2.29-3.92] & $1.90[0.94-3.81]$ & $2.38[1.22-4.65]$ \\
\hline Shock/pulmonary oedema & $6.92[5.68-8.43]$ & & $14.0[10.3-19.1]$ & \\
\hline Renal failure & $2.07[1.68-2.54]$ & & $1.92[1.27-2.91]$ & \\
\hline CCU/ICU & $0.48[0.28-0.85]$ & $0.42[0.25-0.71]$ & & \\
\hline Catheterization laboratory & & & $2.18[1.01-4.71]$ & $2.21[1.05-4.65]$ \\
\hline University hospital & & & $1.22[0.86-1.73]$ & $1.33[0.96-1.84]$ \\
\hline \multicolumn{5}{|l|}{ Internal cross-validation } \\
\hline C-index $[95 \% \mathrm{CI}]$ & $0.819[0.805,0.834]$ & $0.773[0.757,0.788]$ & $0.812[0.785,0.840]$ & $0.800[0.775,0.826]$ \\
\hline CCC\% [95\% CI] & $65.2[49.6,75.6]$ & $63.1[48.7,73.5]$ & $58.3[48.0,64.3]$ & $56.8[47.3,64.3]$ \\
\hline CCC upper reference value\% & 85.3 & 84.3 & 72.6 & 71.7 \\
\hline \multicolumn{5}{|l|}{ External validation } \\
\hline C-index [95\% CI] & $0.807[0.800,0.814]$ & $0.751[0.744,0.759]$ & $0.745[0.727,0.762]$ & $0.736[0.720,0.753]$ \\
\hline CCC\% $[95 \% \mathrm{CI}]$ & $53.6[35.5,61.8]$ & $50.9[37.5,61.1]$ & $47.8[14.9,56.1]$ & $42.1[22.8,52.9]$ \\
\hline CCC upper reference value\% & 92.1 & 91.8 & 81.2 & 75.7 \\
\hline
\end{tabular}

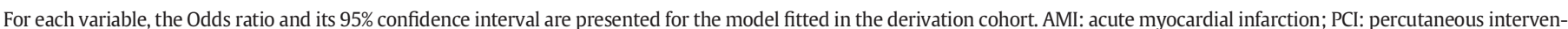

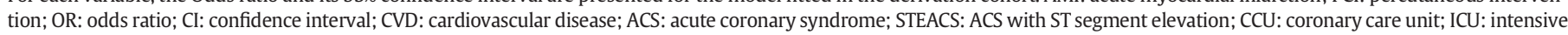
care unit; CCC: concordance correlation coefficient. 
CCU/ICU (Table 3). The clinical model included also cardiogenic shock/ acute pulmonary oedema and renal failure on admission, while the administrative model included previous history of CV disease instead. Age, diabetes, ACS type, cardiogenic shock/acute pulmonary oedema and renal failure on admission were positively associated with in-hospital mortality. Hypertension and presence of CCU/ICU were negatively associated with the outcome.

As for PCI, both the administrative and clinical models included the following predictor variables: diabetes, past history of CV disease, ACS type, catheterization laboratory and university hospital (Table 3). The clinical model included also cardiogenic shock/acute pulmonary oedema and renal failure on admission, while the administrative model included age and hypertension instead. All variables, except for hypertension, were positively associated with in-hospital mortality.

Most of the variables included in the AMI and PCI models had a significant or borderline association with in-hospital mortality at the patient level. In the clinical models, the variable with a larger effect on in-hospital mortality was cardiogenic shock/pulmonary oedema on admission; in the administrative models, it was ACS type.

\subsection{Model validation}

Internal cross-validation showed good discrimination at the patient level of AMI models (c-statistic $=0.819$ and 0.773 for clinical and administrative models, respectively) and PCI models (c-statistic $=0.812$ and 0.800 , respectively) (Table 3). At the hospital level, models showed robust calibration (CCC/upper reference $=76.4 \%$ and $74.9 \%$, for $\mathrm{AMI}$ clinical and administrative models, respectively; $\mathrm{CCC} /$ upper reference $=$ 80.3\% and $79.2 \%$, for PCI models, respectively) (Table 3, Fig. 1).

Performance was also analysed assuming that in-hospital mortality rates were predicted for hospitals located in countries not included in the sample. In this scenario, model discrimination was satisfactory for AMI clinical and administrative models (c-statistic $=0.800$ and 0.747 , respectively) as well as for PCI models (c-statistic $=0.771$ and 0.771 ,
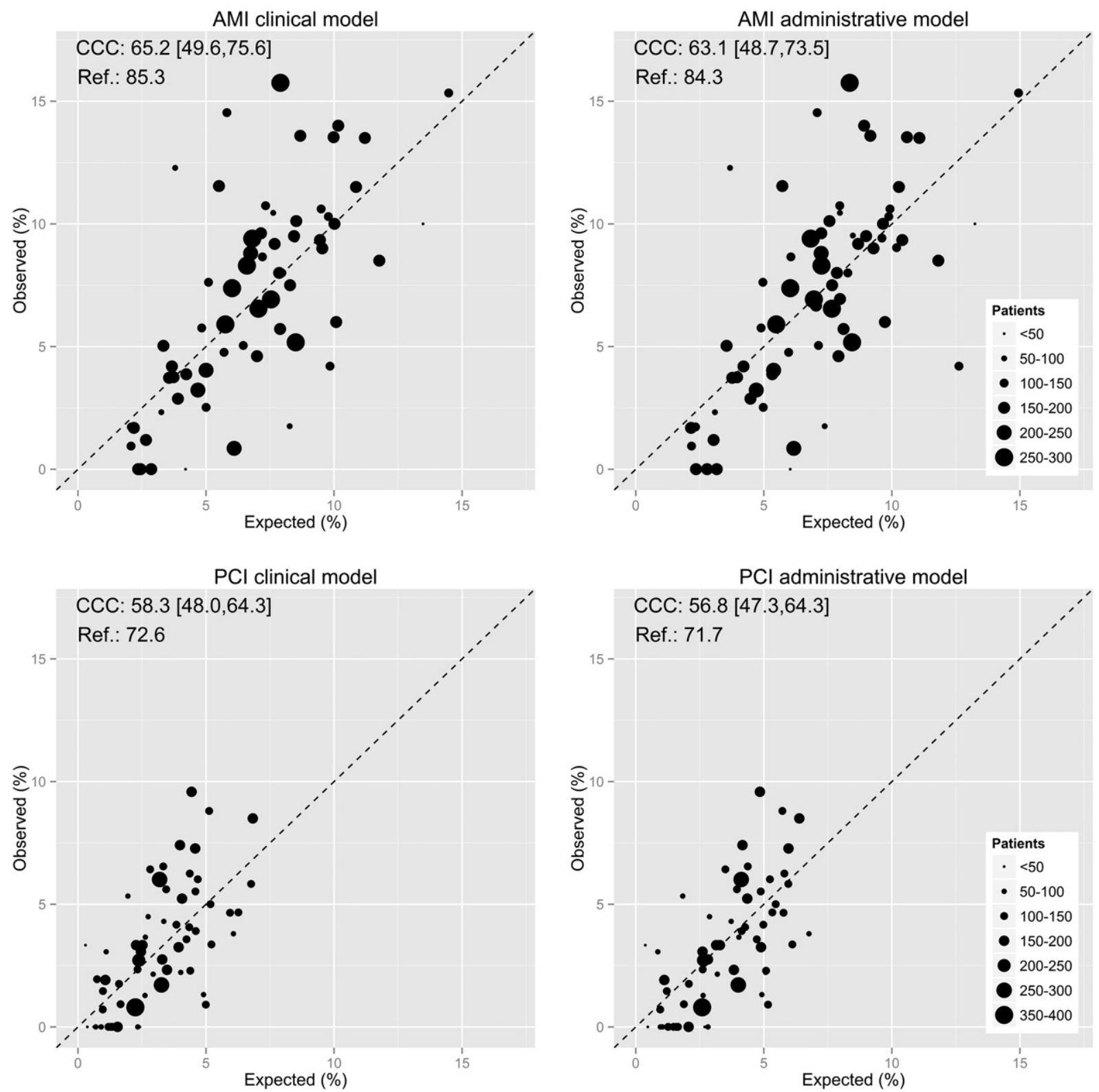

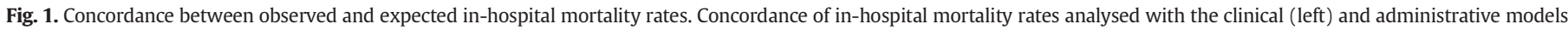

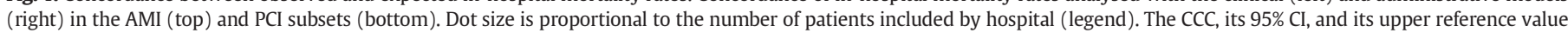
(Ref.) are provided for each scenario. 
respectively). Model calibration, however, was weak in both AMI and PCI models (CCC/upper reference $=21 \%$ for AMI models, and $15 \%$ and $23 \%$, for $\mathrm{PCI}$ clinical and administrative models, respectively).

External validation was performed with the validation cohort summarized in Table 1. The proportion of patients with cardiovascular risk factors, and with renal failure and killip classes III-IV on admission, was lower in the validation cohort compared to the derivation cohort (Table 1). In AMI models, all explanatory variables showed similar ORs in the derivation and validation cohort, except for hypertension and CCU/ICU. However, in PCI models, ORs differed in both cohorts for hypertension, diabetes, past history of cardiovascular disease, ACS type and catheterization laboratory (Tables 1 and S3). Model discrimination was good in the validation cohort (c-statistic $=0.736-0.807$ ), and did not differ between the derivation and the validation cohort except for PCI models (Table 3). Model calibration was acceptable in the validation cohort (CCC/upper reference $=55.4-58.9 \%$ ) and did not differ between the derivation and the validation cohort (Table 3 and Fig. S5).

\subsection{Concordance between administrative and clinical models}

The mean in-hospital MR multiplied by the crude in-hospital mortality was $7.58 \%$ ( $S D=1.50$ ) for patients diagnosed with AMI and $3.23 \%$ $(\mathrm{SD}=0.84)$ for patients who underwent PCI. The concordance between the log-MRs obtained from administrative and clinical models was excellent for AMI and PCI models (97.5\% and 91.6\%, respectively) (Fig. 2).

\subsection{Sensitivity analysis}

Model re-fitting in a subsample excluding transfers and hospital stays $\leq 1$ day yielded similar ORs for all explanatory variables at the patient level, except for diabetes in the PCI administrative model (Tables 3 and S2). Regarding hospital level variables, neither CCU/ICU had an effect on in-hospital mortality in the AMI clinical model, nor catheterization laboratory in the PCI models. No difference was observed in model discrimination and calibration in AMI models. On the other hand, PCI models showed similar discrimination but lower calibration ( $\mathrm{CCC} /$ reference $=$ $59.4 \%$ and $61.2 \%$, for clinical and administrative models, respectively) (Table S2, Fig. S4). Importantly, there was a high concordance between the log-MRs obtained in the whole sample and in the sensitivity analysis subsample: $82.7 \%$ and $80.9 \%$ for AMI clinical and administrative models, and $85.4 \%$ and $84.3 \%$ for PCI clinical and administrative models (Fig. 3 ).

\subsection{Computer-based tool}

The developed models were implemented as an on-line application to provide a straightforward tool to compare AMI/PCI in-hospital mortality rates from cardiology units and hospitals with others. The online application can be accessed from the EURHOBOP website [11], by clicking on the Hospital benchmarking icon. Once in the application, a description of the tool is provided and a page for data entry appears. After sending the data the application produces the percentile distribution of the expected in-hospital mortality rates in all EURHOBOP hospitals with similar characteristics and the location of the assessed hospital in this distribution (Fig. 4).

\section{Discussion}

We developed a benchmarking system to compare performance of European hospitals and cardiology units in AMI management and PCI use. Our system is based on in-hospital mortality predicted at the hospital level by multilevel logistic regression models. The developed models allow cardiologists and other professionals in the cardiology field to compare their own data with others with similar characteristics even if only administrative data is available.

Patient-level variables included in our models were also retained in previous models with similar effects [5-10]. In addition, prior AMI and
PCI models included more comorbidities, admission and procedural variables [5-8], which were not included in our models due to nonavailability, presence of missing values or non-compliance with the reference time. Regarding hospital-level variables, only the AMI model by Seghieri et al. included one, catheterization laboratory [6], which did not contribute significantly to our AMI models, even if CCU/ICU was removed from the list of candidate variables. However, catheterization laboratory was the main hospital-level variable in our PCI models. CCU/ICU was negatively associated with in-hospital mortality in our AMI models, while catheterization laboratory was positively associated with in-hospital mortality in our PCI models. This apparent discrepancy is probably showing - through the CCU/ICU variable - the positive correlation of larger and better-equipped hospitals with in-hospital mortality [18]. At the same time, catheterization laboratory would be representing the negative correlation between time to reperfusion and
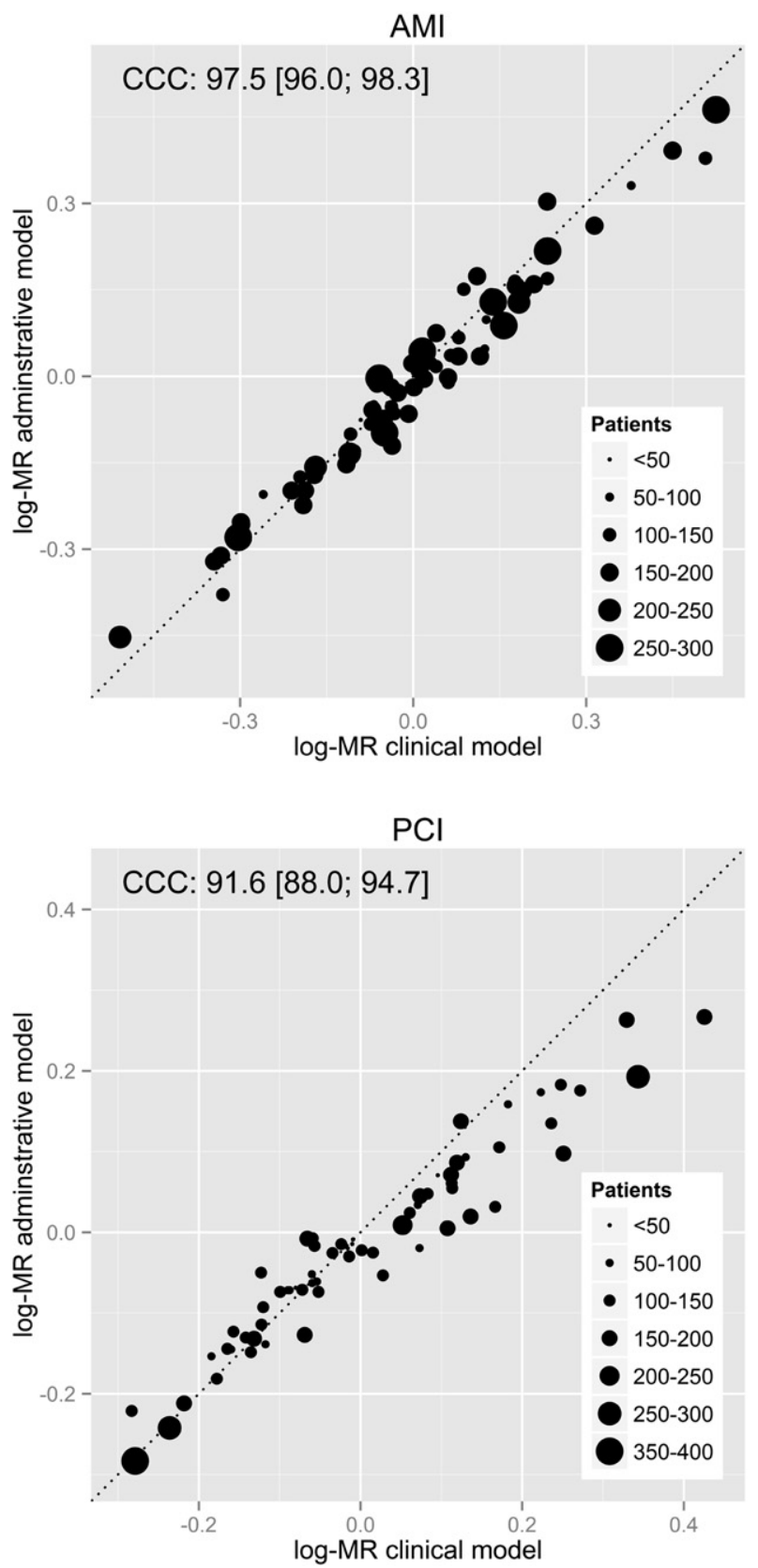

Fig. 2. Concordance between hospital MRs obtained with administrative and clinical models. Concordance of log-MRs analysed in the AMI (top) and PCI subsets (bottom). Dot size is proportional to the number of patients included by hospital (legend). The CCC and its $95 \% \mathrm{CI}$ are provided for each subset. 
in-hospital mortality [19,20], as its effect disappeared when transferred patients were excluded.

Model discrimination at the patient level was similar between our models and those reported in previous studies [5-10]. Our AMI models showed a C-index of 0.77 and 0.82 for administrative and clinical models, respectively. In previous studies, discrimination of AMI models ranged from 0.70 to 0.83 for administrative models and from 0.77 to 0.84 for clinical models [5-8]. As for PCI, the C-index of our clinical model was 0.81 , similar to the discrimination capacity of the only $\mathrm{PCI}$ model - clinical - for hospital benchmarking to date (0.84) [10].

The obtained MRs were consistent with previous studies: a mean of 7.58\% for AMI patients compared to AMI in-hospital and 30-day inhospital mortality rates of $8.10 \%$ and $7.99 \%$, respectively [6,7]. As for PCI, Curtis et al. found an SMR of 1.55\%, compared to the 3.23\% MR observed in this study [10]; this difference is probably due to the exclusion of same-day discharges in the Curtis et al. study.
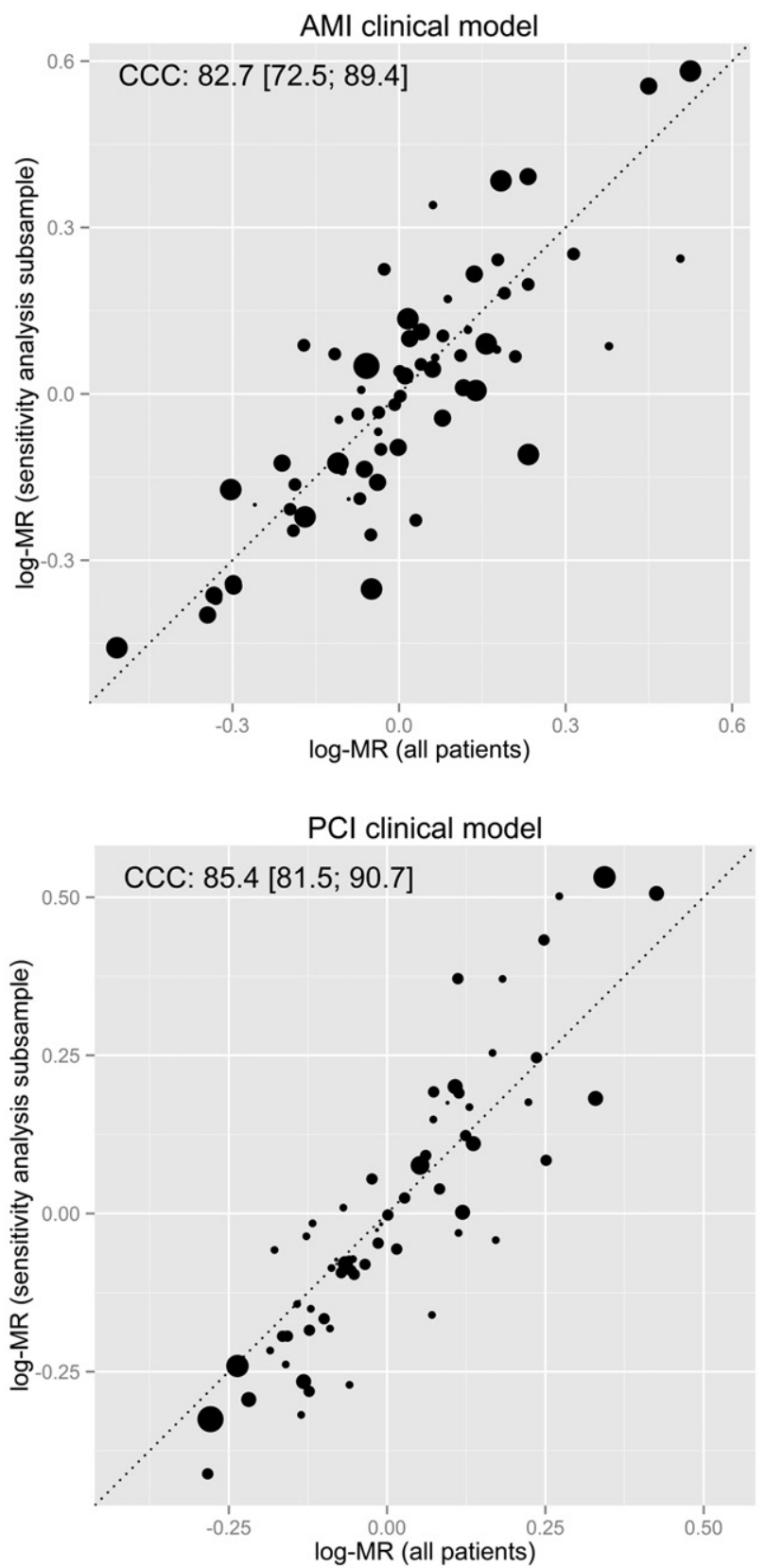

It has been suggested that non-standardized periods of assessment, such as the hospitalization interval, may result in a biased evaluation of hospital performance due to varying lengths of stay (LOS) and transfer of patients [13]. Mean LOS in AMI patients is positively correlated with in-hospital SMR [21], while inter-hospital transfers of AMI patients affects 30-day SMR in acute-care hospitals [22] but not in nonprocedure hospitals [23]. On the other hand, it has been argued that hospitals are only responsible for events that occur within their institution [24]. In this study, we found that $19.6 \%$ to $25.7 \%$ of the patients were transferred and $5.5 \%$ to $8.2 \%$ of patients had LOS $\leq 1$ day. In contrast to the current assumption, our study showed good concordance (81\%$86 \%$ ) between in-hospital log-MR when transfers and short LOS were excluded or not, indicating that these factors do not bias hospital performance assessment based on in-hospital mortality when the appropriate statistical methodology is used. In addition, excluding transfers may cause an inaccurate estimation of quality of care in certain hospitals/
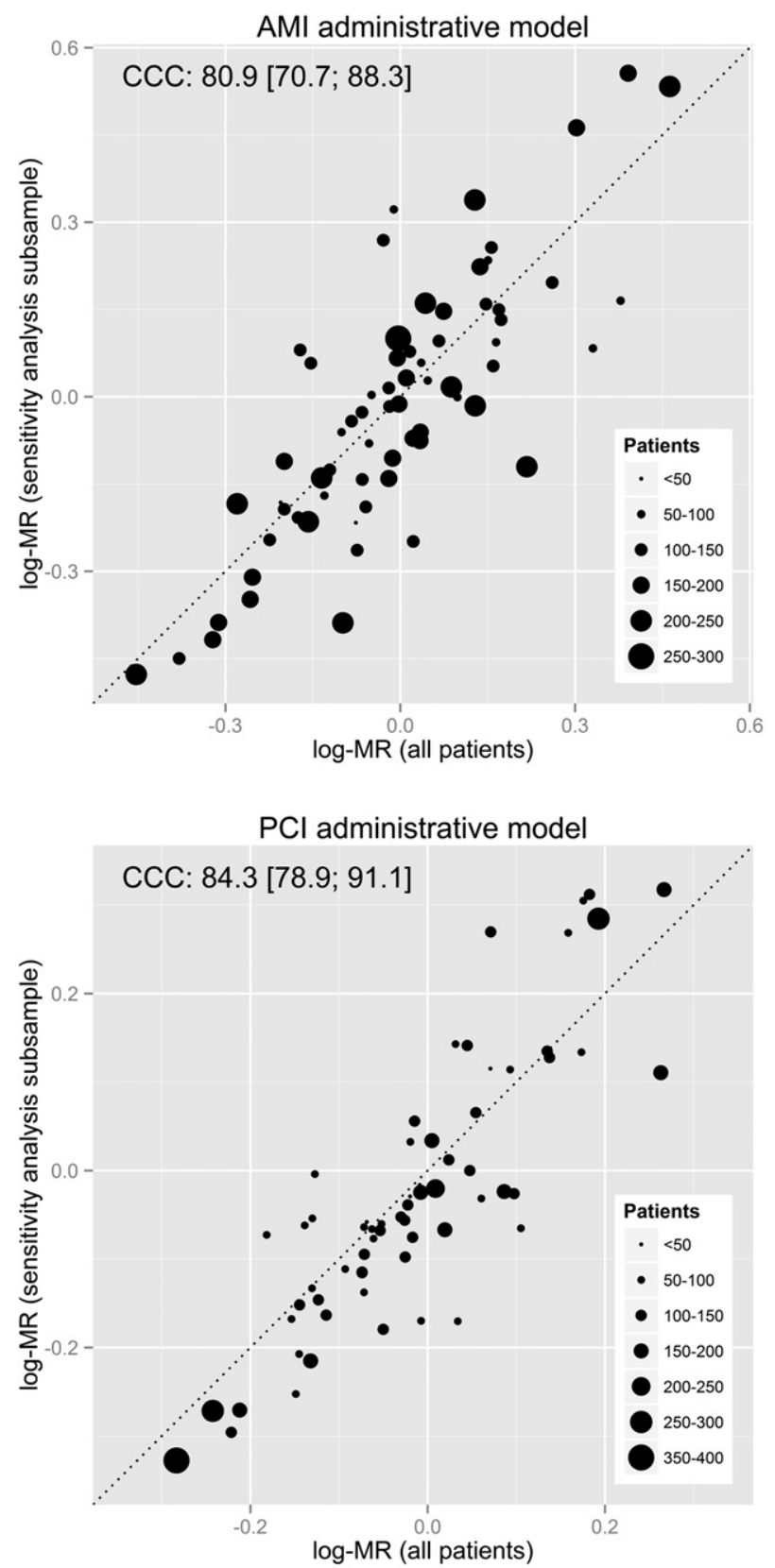

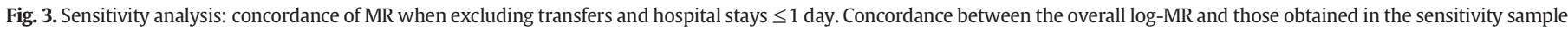

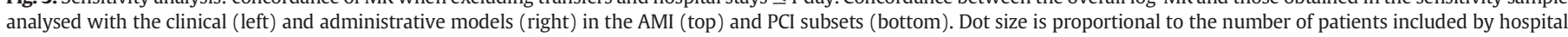
(legend). The CCC and its $95 \% \mathrm{Cl}$ are provided for each scenario. 


\section{Benchmark Results}

\section{- Input data: (click to expand this section)}

How to interpret the benchmarking functions

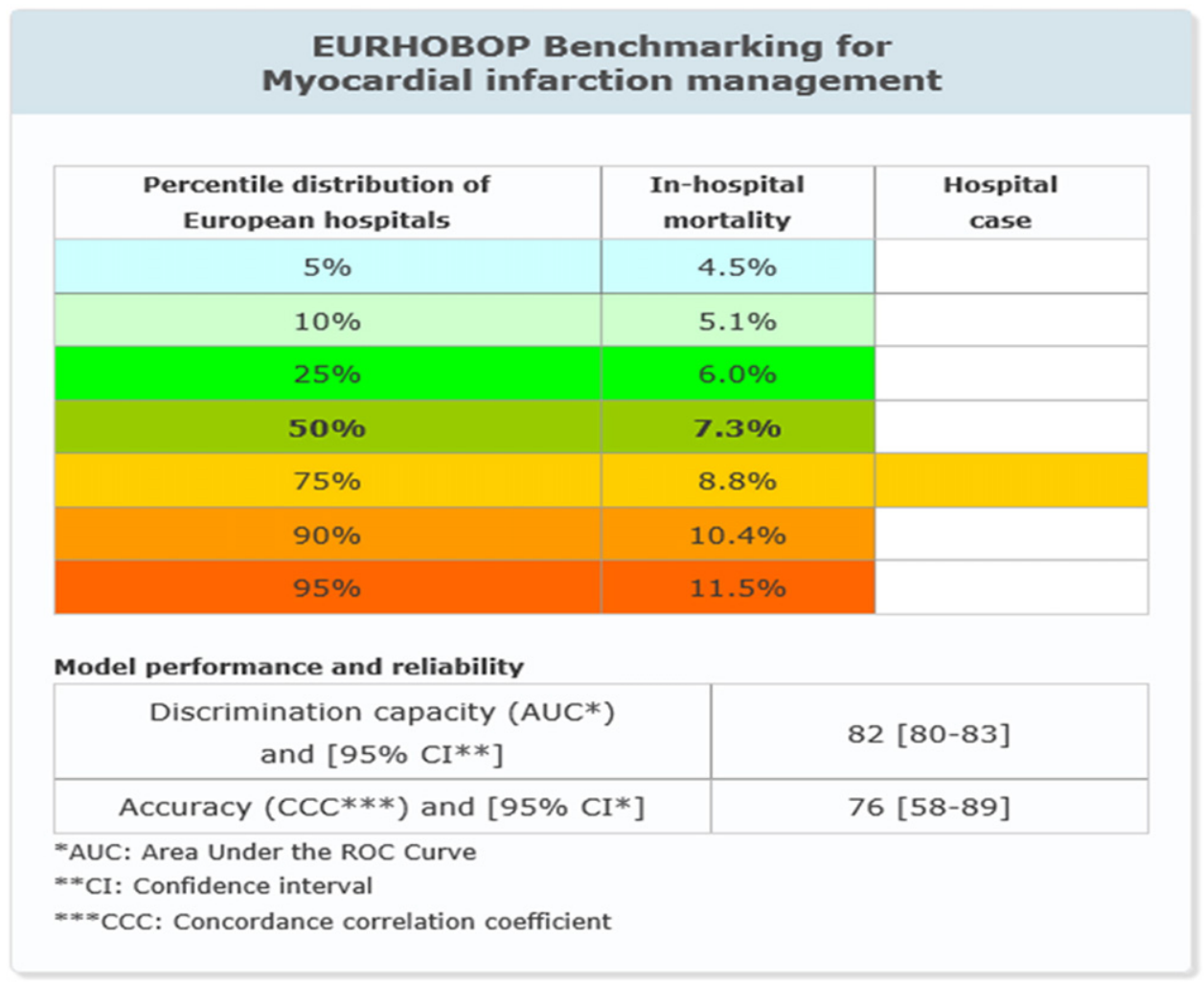

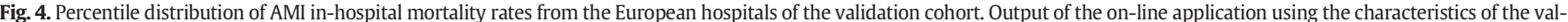

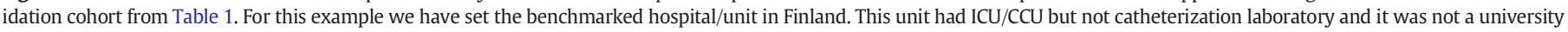

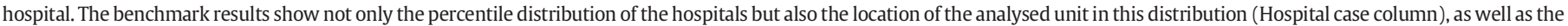
performance measures of the model.

cardiology units [21]. Thus, it may not be necessary to exclude transfers and short LOS when comparing in-hospital mortality from clinical cardiology practice.

Administrative data, which contain important predictors of mortality [25], are often used for outcomes research. Advantages of administrative data are its availability and reduced cost compared to clinical data. On the other hand, administrative data may lack certain predictors of mortality [7]. Two studies demonstrated good correlation between administrative and clinical models for hospital profiling in AMI [5,7]. A more appropriate measure to compare administrative and clinical model is the concordance of their estimates. In this study, we found an excellent concordance ( $>90 \%$ ) between log-MRs obtained with the administrative and clinical models, especially for AMI (97\%). Our administrative models may perform better than previous models due to the model development strategy and to the inclusion of the ACS-type flag [9]. These results show that using the EURHOBOP models, an accurate comparison of in-hospital mortality can be performed by either cardiologists or other healthcare professionals provided that at least administrative data is available.

The available AMI and PCI models for hospital benchmarking are intended for public reporting, and assume access to administrative/ clinical data as well as linkage to mortality registries [5-10]. Our benchmarking system, however, has been designed for cardiologists and healthcare professionals directly involved in the improvement of ACS protocols and patient management. Thus, it has been designed as a tool to improve management of AMI and $\mathrm{PCI}$ patients, through the comparison of in-hospital mortality rates to the rates from similar hospitals. This comparison can be performed anonymously, in a few minutes, and by different users ranging from cardiologists to hospital managers and other stakeholders, as the only requirement is aggregate data from a sample of ACS patients and there is the possibility of applying administrative/ clinical models depending on variable availability.

Our study has several strengths. We developed the first crossEuropean multinational benchmarking models to estimate AMI and PCI in-hospital mortality rates using up-to-date data. We did not exclude patients due to age, LOS, or whether they were transferred or not, which allows more representative estimates of in-hospital mortality rates. We used standard methods for variable selection and statistical analysis, including model derivation and validation. We suggested a metric to evaluate model calibration at the hospital level using the concordance correlation coefficient. And finally, we developed an on-line application to obtain the expected distribution of in-hospital mortality rates from similar hospitals and the location of the assessed hospital/unit in this distribution. This application is a simple, rapid, and precise tool that can be employed by a variety of users to gather insight into the comparison of AMI and PCI in-hospital mortality rates between similar hospitals. 


\section{Study limitations}

The main limitation of our study is the difference between the derivation and the validation cohort. The derivation cohort included ACS patients from 7 countries, while the validation cohort included patients from 3 of these countries. In addition, both cohorts were significantly different regarding the number of patients per hospital and the characteristics of the patients. Despite these differences, the proposed models showed good discrimination and acceptable calibration capacity, suggesting that the models perform adequately even if the sample is completely different from the derivation cohort. Another limitation relates to potential missing variables that could impact in-hospital mortality rates and variability at the hospital- and country-level, such as pharmacological treatment in the acute phase. This limitation is related to the exclusion criteria used and to the goal of providing a simple and precise tool for hospital benchmarking. To fulfil this goal, we had to balance model parsimony and performance. Finally, we observed that the CCC was much lower than expected when the country was simulated as outside the EURHOBOP cohort. The validity of the developed models in countries other than those included in the study merits additional research.

\section{Conclusions}

We present a benchmarking system to assess European hospital performance in AMI and $\mathrm{PCI}$, based on predicted in-hospital mortality rates. Our models take into account hospital and patient characteristics, as well as the hospitals' country. Our system allows comparison of inhospital mortality rates between European hospitals sharing similar patient and hospital characteristics, which could be used by cardiologists to improve their own practices, by hospitals and in a common public health strategy to improve ACS quality of care in Europe.

Supplementary data to this article can be found online at http://dx. doi.org/10.1016/j.ijcard.2015.01.019.

\section{Conflict of interest}

During the conduct of the study, all authors were beneficiary of a grant from the European Commission (SANCO EACH EURHOBOP 2008/1312). IRD, IS, MG, JV, RE and JM received grants from the Health Institute Carlos III-FEDER. IRD received a grant from the Catalan Agency for Management of University and Research Grants. JL received personal fees from BAYER, ASTRA ZENECA and MENARINI outside the submitted work.

\section{Acknowledgements}

This work was supported by the SANCO EAHC grants (EUPHORIC 2003/134 and EURHOBOP 2008/1312), the Health Institute Carlos IIIFEDER (HERACLES Spanish Cardiovascular Research Network RD06/ 0009 and RD12/0042, CB06/02/0029 and CP12/03287 to MG) and the Catalan Agency for Management of University and Research Grants (2005SGR00577, 2009SGR1195 and 2014SGR240, and 2011BP-B00169 postdoctoral contract to IRD - Beatriu de Pinós Program co-financed by the European Commission.

The authors thank Yolanda Ferrer for administrative support, Susana Tello for CRF preparation, Elaine Lilly, PhD, for the revision of the English text, and Mascia Masciocchi, Marco Constantini, and Andrea Paladin for the EURHOBOP website management and web implementation of the developed functions.

\section{References}

[1] J.A. Spertus, M.J. Radford, N.R. Every, E.F. Ellerbeck, E.D. Peterson, H.M. Krumholz Challenges and opportunities in quantifying the quality of care for acute myocardial infarction: summary from the Acute Myocardial Infarction Working Group of the American Heart Association/American College of Cardiology First Scientific Forum on Quality of Care and Outcomes Research in Cardiovascular Disease and Stroke, Circulation 107 (2003) 1681-1691.

[2] Quality of Care and Outcomes Research in CVD and Stroke Working Groups*, Measuring and improving quality of care: a report from the American Heart Association/ American College of Cardiology First Scientific Forum on Assessment of Healthcare Quality in Cardiovascular Disease and Stroke, Circulation 101 (2000) 1483-1493.

[3] A. Donavenian, The role of outcomes in quality assessment and assurance, QRB Qual. Rev. Bull. 11 (1992) 356-360.

[4] J. Tu, L. Khalid, L.R. Donovan, D.T. Ko, Indicators of quality of care for patients with acute myocardial infarction, CMAJ 179 (2008) 909-915.

[5] H.M. Krumholz, Y. Wang, J.A. Mattera, et al., An administrative claims model suitable for profiling hospital performance based on 30-day mortality rates among patients with an acute myocardial infarction, Circulation 113 (2006) 1683-1692.

[6] C. Seghieri, S. Mimmi, J. Lenzi, M.P. Fantini, 30-day in-hospital mortality after acute myocardial infarction in Tuscany (Italy): an observational study using hospital discharge data, BMC Med. Res. Methodol. 12 (2012) 170.

[7] W.E. Weller, G. Kabra, K.S. Cozzens, E.L. Hannan, Appending limited clinical data to an administrative database for assessing institutional quality outliers for AMI care: does it matter? Int. J. Cardiol. 140 (2010) 95-101.

[8] J.S. Ross, C. Maynard, H.M. Krumholz, et al., Use of administrative claims models to assess 30-day mortality among Veterans Health Administration hospitals, Med. Care 48 (2010) 652-658.

[9] A. Bottle, R.D. Sanders, A. Mozid, P. Aylin, Provider profiling models for acute coronary syndrome mortality using administrative data, Int. J. Cardiol. 168 (2013) 338-343.

[10] J.P. Curtis, L.L. Geary, Y. Wang, et al., Development of 2 registry-based risk models suitable for characterizing hospital performance on 30-day all-cause mortality rates among patients undergoing percutaneous coronary intervention, Circ. Cardiovasc. Qual. Outcomes 5 (2012) 628-637.

[11] The EURHOBOP Project, http://www.eurhobop.eu (28th December 2014).

[12] R. André, V. Bongard, R. Elosua, et al., International differences in acute coronary syndrome patients' baseline characteristics, clinical management and outcomes in Western Europe: the EURHOBOP study, Heart 100 (2014) 1201-1207.

[13] H.M. Krumholz, R.G. Brindis, J.E. Brush, et al., Standards for statistical models used for public reporting of health outcomes: an American Heart Association Scientific Statement from the Quality of Care and Outcomes Research Interdisciplinary Writing Group: cosponsored by the Council of Epidemiology and Prevention and the Stroke Council. Endorsed by the American College of Cardiology Foundation, Circulation 113 (2006) 456-462.

[14] K. Burkhardt, I. Kirchberger, M. Heier, et al., Hyponatremia on admission to hospital is associated with increased long-term risk of mortality in survivors of myocardial infarction, Eur. J. Prev. Cardiol. (Nov 11 2014) (pii: 2047487314557963, Epub ahead of print)

[15] P. Colais, M. Di Martino, D. Fusco, et al., Using clinical variables and drug prescription data to control for confounding in outcome comparisons between hospitals, BMC Health Serv. Res. 14 (Oct 23 2014) 495.

[16] I. Ferreira-González, G. Permanyer-Miralda, M. Heras, MASCARA study group, et al. Patterns of use and effectiveness of early invasive strategy in non-ST-segment elevation acute coronary syndromes: an assessment by propensity score, Am. Heart J. 156 (5) (2008) 946-953 (953. e2).

[17] R Core Team, R: A Language and Environment for Statistical Computing, R Foundation for Statistical Computing, Vienna, Austria, 2014. (URL http://www.R-project. org/).

[18] V. Bertomeu, A. Cequier, J.L. Bernal, et al., In-hospital mortality due to acute myocardial infarction. Relevance of type of hospital and care provided. RECALCAR Study, Rev. Esp. Cardiol. 66 (2013) 935-942.

[19] C.P. Cannon, C.M. Gibson, C.T. Lambrew, et al., Relationship of symptom-onset-toballoon time and door-to-balloon time with mortality in patients undergoing angioplasty for acute myocardial infarction, JAMA 283 (2000) 2941-2947.

20] D.S. Pinto, A.J. Kirtane, B.K. Nallamothu, et al., Hospital delays in reperfusion for STelevation myocardial infarction. Implications when selecting a reperfusion strategy, Circulation 114 (2006) 2019-2025

[21] E.E. Drye, S.L. Normand, Y. Wang, et al., Comparison of hospital risk-standardized mortality rates calculated by using in-hospital and 30-day models: an observational study with implications for hospital profiling, Ann. Intern. Med. 156 (2012) 19-26.

[22] M. Kosseim, N.E. Mayo, S. Scott, et al., Ranking hospitals according to acute myocardial infarction mortality: should transfers be included? Med. Care 44 (2006) 664-670.

[23] S.F. Jenks, D.K. Williams, T.L. Kay, Assessing hospital-associated deaths from discharge data. The role of length of stay and comorbidities, JAMA 260 (1988) 2240-2246.

[24] J.A. Barreto-Filho, Y. Wang, S.S. Rathore, et al., Transfer rates from nonprocedure hospitals after initial admission and outcomes among elderly patients with acute myocardial infarction, JAMA Intern. Med. 174 (2014) 213-222.

[25] P. Aylin, A. Bottle, A. Majeed, Use of administrative data or clinical databases as predictors of risk of death in hospital: comparison of models, BMJ 334 (2007) 1044. 